\title{
Extreme Ultraviolet Surface Modification of Polyethylene Terephthalate (PET) for Surface Structuring and Wettability Control
}

\author{
I.U. AhaD ${ }^{a, b *}$, H. Fiedorowicz ${ }^{a}$, B. BudneR ${ }^{a}$, T.J. KAldonski ${ }^{c}$, M. VÁzQUez $^{b}$, \\ A. BARTNIK ${ }^{a}$ AND D. BRABAZON ${ }^{b}$ \\ ${ }^{a}$ Institute of Optoelectronics, Military University of Technology, S. Kaliskiego 2, 00-908 Warsaw, Poland \\ ${ }^{b}$ Advanced Processing Technology Research Centre, School of Mechanical and Manufacturing Engineering, Faculty \\ of Engineering and Computing, Dublin City University, Dublin 9, Ireland \\ ${ }^{c}$ Faculty of Mechanical Engineering, Military University of Technology, Warsaw, Poland
}

\begin{abstract}
The surface modification of polyethylene terephthalate (PET) polymer films has been performed by irradiation of extreme ultraviolet photons to investigate the effect of surface structuring on wettability control. For biomedical engineering applications, surface structuring and wettability control of PET films could enhance the polymer biocompatibility by promoting cell adhesion and consequently proliferation. The PET films are irradiated with laser plasma extreme ultraviolet source based on double stream gas puff target under different environments. The extreme ultraviolet modified PET film surfaces are characterized by atomic force microscopy and WCA goniometer. The extreme ultraviolet surface modification resulted in the formation of nano- and microstructuring on the polymer surfaces. The surface structuring consequently increased WCA making the PET surfaces more hydrophobic. The results demonstrate the direct relationship between surface roughness and hydrophobicity for extreme ultraviolet modified PET samples.
\end{abstract}

DOI: 10.12693/APhysPolA.129.241

PACS: 81.05.Lg, 81.40.Wx, 81.16.Rf, 81.65.-b

\section{Introduction}

PET is widely used in various biomedical engineering applications. The PET films are used for fabrication of scaffolds and vascular grafts, and also used for tissue replacement surgery [1-3]. The surface roughness and wettability often needed to be modified for enhancement in biocompatibility of PET surfaces [3]. However the conventional surface modification tools alter the bulk material mechanical properties [4]. Recent advancements in the laboratory scale laser produced plasma sources provide opportunities to exploit soft X-ray and extreme ultraviolet regions for applications in biomedical engineering [5-9]. The surface structuring of polymers by EUV photons by ablation mechanism has been demonstrated previously. However a general ablation theory is not yet available that could be used to model the physical and chemical modifications induced by EUV ablation [10]. This study is an attempt to correlate the EUV induced surface structuring of PET samples with subsequent changes in wettability. This correlation would allow the fine tuning of surface wettability of polymer samples (such as PET) for specific applications related to biocompatibility control and artificial muscles in biomedical engineering [11-13].

*corresponding author; e-mail: inamul.ahad@dcu.ie
In this study, the EUV surface modification of PET films has been performed to characterize the EUV induced modifications to surface roughness and wetting behavior. The EUV photons have limited penetration depth in polymers (up to $100 \mathrm{~nm}$ ), therefore the surfaces of PET films can be modified efficiently and the bulk mechanical properties could remain intact [3]. The PET surfaces were modified in the presence and absence of reacting gas (nitrogen) to investigate the dominancy of various processes that occurred during laser (in this case EUV) ablation (such as selective ablation, functionalization, and total ablation).

\section{Materials and methods}

The PET films obtained from Goodfellow Cambridge Limited, United Kingdom were used for EUV surface modification. The PET films were cut into $12 \mathrm{~mm} \times$ $12 \mathrm{~mm}$ small pieces and irradiated with a laser-plasma EUV source based on a double-stream gas-puff target, irradiated with the $3 \mathrm{~ns} / 0.8 \mathrm{~J} \mathrm{Nd}$ :YAG laser pulse at $10 \mathrm{~Hz}$. The laser target was constructed by using krypton and xenon mixture as working gas and helium as confining gas. The EUV photons emitted from laser plasma were collected and delivered to polymer processing chamber by gold plated grazing incidence ellipsoidal mirror. The EUV radiations were focused onto an $X Y Z$ motorized stage in the polymer processing chamber. The sample stage was adjusted to position $3 \mathrm{~mm}$ downstream the EUV focal spot. The fluence of incoming EUV photons 
at this position was about $65 \mathrm{~mJ} / \mathrm{cm}^{2}$ [14]. The polymer samples were mounted on the stage, kept at $3 \mathrm{~mm}$ distance from focal spot and irradiated with 20 or 30 EUV pulses in raster scanning mode. The EUV irradiation was performed in the presence of helium (non reactive) or nitrogen (reacting) environments in separate experiments. The detailed arrangement and working of the source was documented before $[3,6,14]$.

Atomic force microscopy (AFM), supplied by NTMDT, Russia, investigated the morphological modifications induced by EUV surface structuring and the average surface roughness $\left(S_{a}\right)$ was calculated for each sample. The water contact angles (WCA) of pristine and EUV treated polymers were measured using the static sessile drop method by employing KSV Instruments CAM 100. The detailed procedure of AFM imaging and WCA measurements of the EUV modified surfaces was documented before $[6,7,14]$.

\section{Results and discussion}

The topography and the surface roughness of the pristine and EUV modified PET surfaces were investigated by AFM. The PET samples were scanned at a distance of $1 \mathrm{~mm}$ from the edge of the EUV patterned area towards the center of EUV trace. The PET surfaces modified with EUV irradiation demonstrated pronounced morphological changes. The pristine PET sample was quite smooth. $S_{a}$ of pure PET sample was $6.6 \mathrm{~nm}$. The EUV irradiation of PET sample with 20 and 30 pulses in the helium environment resulted in the formation of pseudo-conical shaped rippled structures. The surface roughness was increased by EUV irradiation and the measured $S_{a}$ of the PET surfaces irradiated with 20 and 30 EUV pulses was $240 \mathrm{~nm}$ and $271 \mathrm{~nm}$, respectively. The next experiments were performed in the nitrogen environment. $S_{a}$ measured was $215 \mathrm{~nm}$ and $234 \mathrm{~nm}$ for the PET samples irradiated with 20 and 30 EUV pulses, respectively. The morphology was however moderately distinct from the previous case as the pseudo-conical shaped structures were more pronounced making discontinued wall type elongated structures. The difference in the morphology of two experimental conditions is due to the total fluence accumulated by number of EUV pulses that reached PET samples.

The material ablation depends upon various factors such as wavelength, fluence, material sensitivity, heat capacity and number of shots irradiated on the surface [15]. If the fluence is near to ablation threshold, chemical modifications would be the dominating process. In case the fluence cross ablation threshold values, the material ablation would be the dominating process [16]. Therefore after a single EUV shot, one would expect strong chemical modifications and very small changes in material surface morphology. The source used in this study produced EUV photons with maximum intensity around $10 \pm 1 \mathrm{~nm}$ wavelength corresponding to photon energy of $112 \mathrm{eV}$ [3]. Therefore a single EUV photon was able to break several bonds, as the energy of irradiating photons was way above the binding energies of bonds in the polymer chains such as $\mathrm{C}-\mathrm{C}(3.6 \mathrm{eV}), \mathrm{C}-\mathrm{N}(3.2 \mathrm{eV}), \mathrm{C}-\mathrm{O}$ $(3.7 \mathrm{eV}), \mathrm{N}-\mathrm{N}(1.7 \mathrm{eV})$ and $\mathrm{C}-\mathrm{F}(5.0 \mathrm{eV})$. Therefore even with a single shot with $65 \mathrm{~mJ}$ fluence, surface structuring could be obtained but lower material ablation would be expected. As observed from the experiments carried out in this study, the increase in the surface roughness of PET samples was higher in case of EUV irradiation in the helium environment as compared to samples irradiated in the nitrogen environment. Lower increment in the surface roughness could be attributed to the fact that some amount of EUV photons consumed in the ionization of nitrogen gas, thus lower fluence reached the polymer samples. Consequently discontinued wall type structures were obtained. In case of helium environment, the samples received relatively higher cumulative fluence as no ionization was performed by the EUV photons and subsequently higher ablation was obtained. This resulted in formation of wall type rippled structures. The laser ablation not only depend upon the wavelength and energy but also depend upon the repetition rate, the accumulated energy deposited onto the surface layer, and the time of irradiation. Since the experiments were performed with low number of pulses (two seconds and three seconds) the morphology formed in both environments were moderately distinct.

The WCA measurements were acquired to investigate the effect of EUV irradiation on the EUV modified PET surfaces. The WCA of pristine PET sample obtained from Goodfellow UK was about $81.5^{\circ}$. The PET samples irradiated with 20 and $30 \mathrm{EUV}$ in the helium environment exhibit increased $\mathrm{WCA}$ to $95^{\circ}$ and $98^{\circ}$, respectively. The WCA of PET samples irradiated with 20 and 30 EUV pulses in the nitrogen environment were $88^{\circ}$ and $91^{\circ}$, respectively. The results are summarized in Table I. $S_{a}$ and the wettability results are normalized to $0-1$ scale and presented in Fig. 1. Although the surface roughness and wettability are two separate quantities with distinct units and scales, the normalization of the results was performed to visualize the relationship of these two properties by EUV irradiation in different environment. Such graphical visualization would help to understand the effect of EUV induced structuring on the wettability of PET surfaces.

\section{TABLE I}

Summarized surface roughness and wettability results of pure and EUV modified PET samples.

\begin{tabular}{c|c|c|c}
\hline \hline environment & $n$ & $S_{a}[\mathrm{~nm}]$ & WCA [degrees] \\
\hline \multicolumn{2}{c|}{ pristine PET sample } & 6.6 & 81.5 \\
\hline \multirow{2}{*}{ Helium } & 20 & 240 & 95 \\
\cline { 2 - 4 } & 30 & 271 & 98 \\
\hline \multirow{2}{*}{ Nitrogen } & 20 & 215 & 88 \\
\cline { 2 - 4 } & 30 & 234 & 91
\end{tabular}




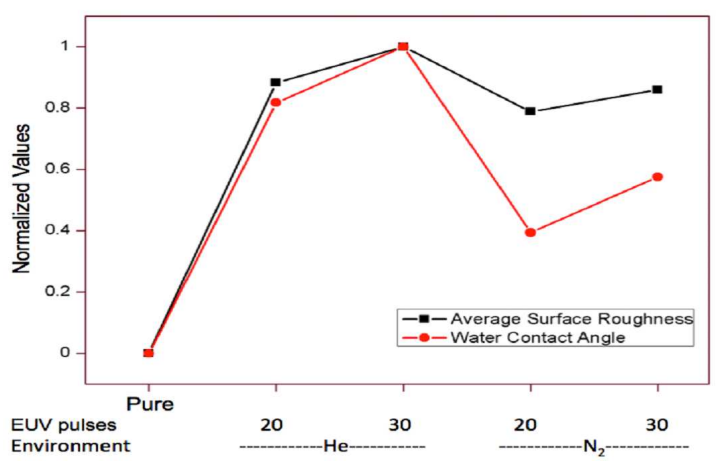

Fig. 1. Normalized $S_{a}$ and WCA of pristine and after $n$ EUV shots modified surfaces.

The EUV irradiation in the helium environment resulted in dominancy of PET surface ablation, subsequently strong morphological modifications and high hydrophobicity was registered. The introduction of nitrogen gas reduced the EUV fluence at polymer processing site due to absorption of EUV photons for nitrogen ionization. Therefore the chemical modifications could be the dominant process. The wall type surface structuring of PET films could be interesting to use for fabrication of lab-on-a-chip (LOC) devices for microfluidic applications.

\section{Conclusion}

The increment in the EUV pulses resulted in increment of WCA in all experimental setups due to increase in the surface roughness. However, the introduction of nitrogen atoms in the PET films tends to reduce the WCA, that might be due to introduction of nitrogen containing functional groups on polymer surface. Further studies on chemical modifications will be performed to investigate the effect of nitrogen incorporation into PET surfaces with respect to surface morphology.

\section{Acknowledgments}

Authors acknowledges financial support from the EU FP7 Erasmus Mundus Joint Doctorate Programme EXTATIC under framework partnership agreement FPA2012-0033. With support from the 7th Framework Programme Laserlab Europe project (No. 284464). This work supported by a research grant from Science Foundation Ireland (SFI) under Grant No. 12/IA/1576.

\section{References}

[1] B.L. Seal, T.C. Otero, A. Panitch, Mater. Sci. Eng. $R$ Rep. 34, 147 (2001).

[2] L.G. Griffith, Acta Mater. 48, 263 (2000).

[3] I.U. Ahad, A. Bartnik, H. Fiedorowicz, J. Kostecki, B. Korczyc, T. Ciach, D. Brabazon, J. Biomed. Mater. Res. Part A 102, 3298 (2014).

[4] J. Heitz, V. Svorcík, L. Bacáková, K. Rocková, E. Ratajová, T. Gumpenberger, D. Bäuerle, B. Dvoránková, H. Kahr, I. Graz, C. Romanin, J. Biomed. Mater. Res. A 67, 130 (2003).

[5] P. Wachulak, A. Torrisi, M.F. Nawaz, A. Bartnik, D. Adjei, Š. Vondrová, J. Turňová, A. Jančarek, J. Limpouch, M. Vrbová, H. Fiedorowicz, Microsc. Microanal. 21, 1214 (2015).

[6] I.U. Ahad, B. Butruk, M. Ayele, B. Budner, A. Bartnik, H. Fiedorowicz, T. Ciach, D. Brabazon, Nucl. Instrum. Methods Phys. Res. B 364, 98 (2015).

[7] I.U. Ahad, B. Budner, H. Fiedorowicz, A. Bartnik, D. Brabazon, Eur. Cells Mater. 26, 145 (2013).

[8] H. Fiedorowicz, I.U. Ahad, A. Bartnik, T. Fok, R. Jarocki, B. Korczyc, J. Kostecki, A. Szczurek, M. Szczurek, P. Wachulak, L. Wegrzynski, in: Proc. 2014 Int. Conf. Laser Optics, St. Petersburg (Russia), St. Petersburg 2014.

[9] D. Adjei, M.G. Ayele, P. Wachulak, A. Bartnik, Ł. Wegrzynski, H. Fiedorowicz, L. Vyšín, A. Wiechec, J. Lekki, W.M. Kwiatek, L. Pina, M. Davídková, L. Juha, Nucl. Instrum. Methods Phys. Res. B 364, 27 (2015).

[10] C. Liberatore, A. Bartnik, I.U. Ahad, M. Toufarová, I. Matulková, V. Hájková, L. Vyšín, T. Burian, L. Juha, L. Pina, A. Endo, T. Mocek, Proc. SPIE 9510, EUV and X-ray Optics: Synergy between Laboratory and Space IV, 951011 (2015).

[11] S. Lerouge, A. Major, P.L. Girault Lauriault, M.A. Raymond, P. Laplante, G. Soulez, F. Mwale, M.R. Wertheimer, M.-J. Hébert, Biomaterials 28, 1209 (2007).

[12] D.F. Williams, Biomaterials 29, 2941 (2008).

[13] A.M. Gallardo-Moreno, M.A. Pacha-Olivenza, L. Saldaña, C. Pérez-Giraldo, J.M. Bruque, N. Vilaboa, M.L. González-Martín, Acta Biomater. 5, 181 (2009).

[14] A. Bartnik, H. Fiedorowicz, R. Jarocki, J. Kostecki, M. Szczurek, P.W. Wachulak, Nucl. Instrum. Methods Phys. Res. A 647, 125 (2011).

[15] N. Bityurin, N. Arnold, B. Luk'yanchuk, D. Bäuerle, Appl. Surf. Sci. 127-129, 164 (1998).

[16] R. Srinivasan, B. Braren, Chem. Rev. 89, 1303 (1989). 